\title{
DEM investigation on characteristics of rolling resistance for modelling particle shape
}

\author{
Lunlun Zhou ${ }^{1}$, Xihua $\mathrm{Chu}^{1, *}$, and Yuanjie $\mathrm{Xu}^{1}$ \\ ${ }^{1}$ School of Civil Engineering, Wuhan University, Wuhan, 430072, China
}

\begin{abstract}
To examine the capability of rolling resistance to model the effects of particle shape, two sets of samples, composed of binary clumped particles and circular particles with rolling resistance, are tested in DEM simulation. The coefficient of rolling friction is estimated based on the energy dissipation. The effects of rolling resistance and particle shape on the shear strength, deformation behavior and non-coaxiality are compared. The numerical results show that rolling resistance reproduces well the effect of particle shape on the peak strength. However, other macro-properties, such as residual strength, elasticity modulus, poisson's ratio, dilatancy and non-coaxiality, introduced by rolling resistance both exist certain differences compared with the effect of particle shape. The discrepancies is thought to be due to the increasing compressibility of samples as the particle shape becomes more elongated, which cannot be reproduced by increasing rolling friction.
\end{abstract}

\section{Introduction}

The discrete element method (DEM) has been a valuable tool in the study of mechanical behavior of granular materials. Most DEM studies simulate granular systems as assemblies of 2D disks or 3D spheres. However, real granular materials are almost composed of particles of complicated shapes. And it has been shown that the nonspherical shape of particles has significant effects on shear strength, deformation behavior and microstructural properties of granular systems [1].

One of the major challenges for DEM is to simulate non-spherical particles in an efficient manner. When modeling complicated particle shapes in DEM, it brings more complex geometric algorithm, contact judgment and contact model. All these cause sharply rising in computational cost. Shape is thus an expensive thing to model in DEM. Consequently, rolling resistance is typically used in DEM as a simple way of modeling the effects of irregular particle shape.

In the conventional discrete element formulation, the interactions between two particles consist only of contact forces in the normal and tangential directions. Particles are assumed to rotate freely and offer no resistance to rotation. The technique (using rolling friction to simulate shape) incorporates a rotational frictional torque to inhibit particle rotation. The torques acting on the two particles in contact are usually applied as a couple of action and reaction. The magnitude and direction of this torque depend on the relative rotation between the particles in contact. In reality, the torque produced by particle shape is an eccentric torque caused by the normal contact force, as shown in Figure 1 [2]. The magnitude and direction of this eccentric torque are not relative to the torque acting on the adjacent particle in the contact.

As above, the rolling frictional torque and the real eccentric torque have different action mechanisms on the microscopic level. The question then is what effects of particle shape on macroscopic mechanical behaviors can be captured by using rolling resistance. This question is the primary concern of this paper. In this study, two sets of samples, composed of binary clumped particles and composed of circular particles with rolling resistance, are tested in DEM simulation. The capability of rolling resistance to model the effects of particle shape is examined by comparing the effects of rolling resistance and particle shape on the shear strength, deformation behavior and non-coaxiality of granular materials.

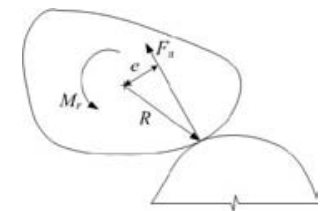

Fig. 1. Contact between particles with irregular shapes [2]

\section{Rolling resistance model}

Many researchers have suggested various contact models incorporating rolling resistance [3-5]. The model used in this study was initially developed by Iwashita and Oda [3] and was identified to be superior in terms of its behavior for static materials [4]. The rolling resistance torque applied to contact is defined in an incremental way by the following

\footnotetext{
Corresponding author: Chuxh@,whu.edu.cn
} 


$$
M_{r, t+\Delta t}=\left\{\begin{array}{l}
M_{r, t}-k_{r} \omega_{r e l} \Delta t \text { provide that } \\
\left|M_{r, t}-k_{r} \omega_{r e l} \Delta t\right|<\mu_{r} R_{r}\left|F_{n}\right|, \\
\mu_{r} R_{r}\left|F_{n}\right| \frac{M_{r, t}-k_{r} \omega_{r e l} \Delta t}{M_{r, t}-k_{r} \omega_{r e l} \Delta} \text { otherwise. }
\end{array}\right.
$$

Where $M_{r, t}$ is the rolling resistance torque at time $t$, $\Delta t$ is the time increment, $\omega_{r e l}$ is the relative angular velocity of the particles in contact, $F_{n}$ is the normal contact force at the contact, $R_{r}=R_{i} R_{j} /\left(R_{i}+R_{j}\right)$ is the common radius, $R_{i}, R_{j}$ is the radius of the two particles in contact respectively, $k_{r}$ is the rolling stiffness and $k_{r} \approx k_{s} R_{r}{ }^{2}$ according to the suggestion of Iwashita and Oda [3], $\mu_{r}$ is the coefficient of rolling friction. This rolling resistance model defines that the rolling resistance torque is not exceeding the maximum value $M_{r, \max }=\mu_{r} R_{r}\left|F_{n}\right|$.

\section{DEM simulation method}

Two sets of DEM simulations are carried out using the DEM software PFC2D. In the first set of simulations, the samples compose of binary clumped particles. The clumped particles consist of two overlapping circular particles of the same radius. And varying relative spacing, $\delta$, is used to characterize different particle shapes, where $\delta$ is the value of the spacing between the centers of the constitutive particles normalized by the radius of the constitutive particles. In the second set, circular particles with rolling resistance are used to simulate the effect of particle shape. Different equivalent rolling frictions, $\mu_{r}$, are estimated corresponding to the different clump shapes.

\subsection{Rolling friction and shape}

The coefficient of rolling friction, $\mu_{r}$, can be estimated in two different ways [1-2]. In the first approach [2], the selection of the coefficient of rolling friction is based on the normalized average contact eccentricity of nonspherical particles, but the DEM result showed that half the rolling friction estimated from the normalized eccentricity is appropriate to capture the similar behavior of the case of non-spherical particles. The second approach[1], used in this study, defined the coefficient of rolling friction based on the energy dissipation associated with relative rotation between particles in contact. And this calculated coefficient is exactly one half of the value calculated by the first approach.

Considering a binary clumped particle and a circular particle with rolling friction roll on a flat surface with a vertical force $F_{n}$ exerted at the center of mass, as shown in Figure 2. The horizontal force $T$ is applied at the center of mass to roll the particles. The work required to roll the binary clumped particle one round is

$$
W_{\mathrm{c}}=\delta R_{l} F_{n} .
$$

Where $R_{l}$ is the radius of the constitutive particles of the clumped particle. In the case of circular particle with rolling friction, the work required is

$$
W_{\mathrm{r}}=2 \pi M_{r, \max }=2 \pi \mu_{r} R_{2} F_{n} .
$$

Where $R_{2}$ is the radius of the clumped particle. Assuming the work required is equal, the coefficient of rolling friction can be given by

$$
\mu_{r}=\delta R_{1} / 2 \pi R_{2}
$$

Assuming that clumped particle and circular particle have the same volume, the relationship between $R_{l}$ and $R_{2}$ is

$$
R_{1} / R_{2}=\sqrt{\pi /\left(2 \pi+\delta / 2 \sqrt{1-\delta^{2} / 4}-2 \operatorname{COS}^{-1}(\delta / 2)\right)} .
$$

For each different clump shape, the equivalent rolling friction is calculated and outlined in Table 1. Other parameters used in DEM simulations are outlined in Table 2.
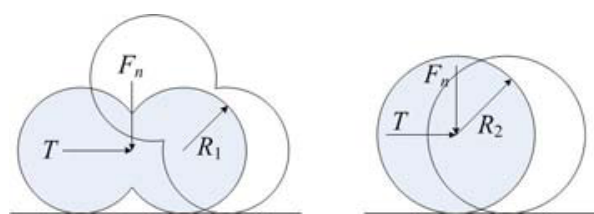

Fig. 2. Rolling of a binary clumped particle and a circular particle with rolling friction on a plane [1].

Table 1. Clump spacing and corresponding rolling frictions.

\begin{tabular}{|c|c|}
\hline Relative spacing $\delta$ & Rolling friction $\mu_{r}$ \\
\hline 0.49 & 0.07 \\
\hline 0.79 & 0.11 \\
\hline 1.10 & 0.14 \\
\hline 1.45 & 0.18 \\
\hline 1.76 & 0.20 \\
\hline
\end{tabular}

Table 2. Parameters used in DEM simulations.

\begin{tabular}{|c|c|}
\hline Particle size: $\mathrm{mm}$ & $0.75-1.0$ \\
\hline Size distribution & Uniform \\
\hline Porosity & 0.14 \\
\hline Particle density: $\mathrm{kg} / \mathrm{m}^{3}$ & 2650 \\
\hline Particle sliding friction & 0.5 \\
\hline Particle normal stiffness: $\mathrm{N} / \mathrm{m}$ & $5.0 \times 10^{8}$ \\
\hline Particle tangential stiffness: $\mathrm{N} / \mathrm{m}$ & $2.5 \times 10^{8}$ \\
\hline Particle rolling stiffness: $\mathrm{N} \cdot \mathrm{m}$ & 500 \\
\hline
\end{tabular}

\subsection{Testing procedure}

Two types of tests, biaxial tests and simple shear test, are conducted to investigate the stress-strain behaviors and non-coaxiality of the two sets of samples.

In the biaxial tests, the samples consist of 5792 particles or clumps, and its initial dimension is $180 \times 90$ $\mathrm{mm}$. Four frictionless walls are created as the rigid boundaries. The stiffness of the horizontal walls is set to be the same as the particles, and the stiffness of vertical walls is one tenth of the stiffness of particles. The samples are first consolidated isotropically with the confining pressure of 1.0 Mpa and then sheared under 
the strain rate of $0.02 \% / \mathrm{min}$ until the axial strain reached $12 \%$. The lateral walls are controlled by a servo-control algorithm to keep the confining pressure constant during shear stage.

In the simple shear tests, the samples consist of 5148 particles or clumps, and its initial dimension is $120 \times 120$ $\mathrm{mm}$. The stiffness and friction coefficient of the wall boundaries are the same as the particles. The samples are first subjected to anisotropic consolidation with the vertical normal stress of 1.2 Mpa and horizontal normal stress of $0.8 \mathrm{Mpa}$. Then the two vertical walls are rotated at a constant angular velocity, $\omega$, of $0.02 \mathrm{rad} / \mathrm{min}$ about the mid-points of the two walls. The two horizontal walls are translated with a compatible horizontal velocity in opposite directions, and the vertical velocities of the horizontal walls are adjusted using a servo-control algorithm to keep the vertical normal stress constant. The test continues until the shear strain, $\gamma=\sin (\omega t)$, reached $25 \%$.

\section{DEM results and analysis}

\subsection{Stress-strain characteristics}

The curves of shear strength, $q=\sigma_{1}-\sigma_{2}$, and volumetric strain, $\varepsilon_{v}$, versus axial strain, $\varepsilon_{l}$, obtained from the biaxial tests of the two sets of the samples are plotted in the Figure 3 and 4, respectively. In the case of clumped particles, the volumetric strain curves decline gradually as the relative spacing increases. The corresponding shear strength curves transforms gradually from strain softening to strain hardening. However, in the case of circular particles with rolling resistance, there are no significant differences between the volumetric strain curves for varying rolling frictions, and the corresponding shear strength curves show distinct strain hardening characteristic for all rolling frictions.

In order to compare the effects of rolling resistance and particle shape in more detail, the macro-parameters for the two sets of smaples obtained from Figure 3 and 4 are outlined in Table 3. The symbol "CP" and "RL" mean the case of clumped particles and circular particles with rolling resistance, respectively. It can be seen that the peak shear strengths, $q_{\max }$, are both increasing with the increasing relative spacing and rolling friction in the two cases, and the values of $q_{\max }$ in the case of clumped particles are coincidence with the data in case of rolling resistance basically. The residual shear strength, $q_{\text {res }}$, increases gradually as the shape becomes less circular, whereas it almost keeps constant as rolling friction increases. The elasticity modulus, $E$, in the case of circular particles with rolling resistance increases with rolling friction and is up to $550 \mathrm{Mpa}$, which is much higher than that of the clumped particles. The poisson's ratio, $v$, of the samples of clumped particles decrease with relative spacing, while the evolution of $v$ in the rolling resistance case is not clear. The values $v$ of former case is generally higher than the latter case. The peak volumetric contraction, $\varepsilon_{v \max }$, increases in the first case, but keeps constant in the latter case, and the dilatancy of the samples of clumped particles is significantly less than that of the circular particles with rolling resistance.
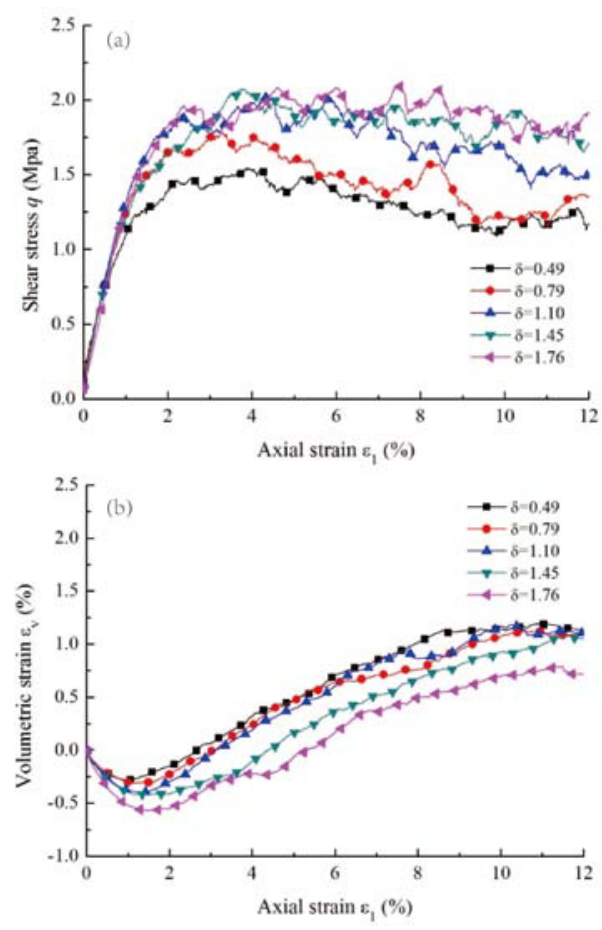

Fig. 3. Stress-strain relations for binary clumped particles of varying relative spacing in biaxial tests: (a) $q$ versus $\varepsilon_{l}$, and (b) $\varepsilon_{v}$ versus $\varepsilon_{l}$.
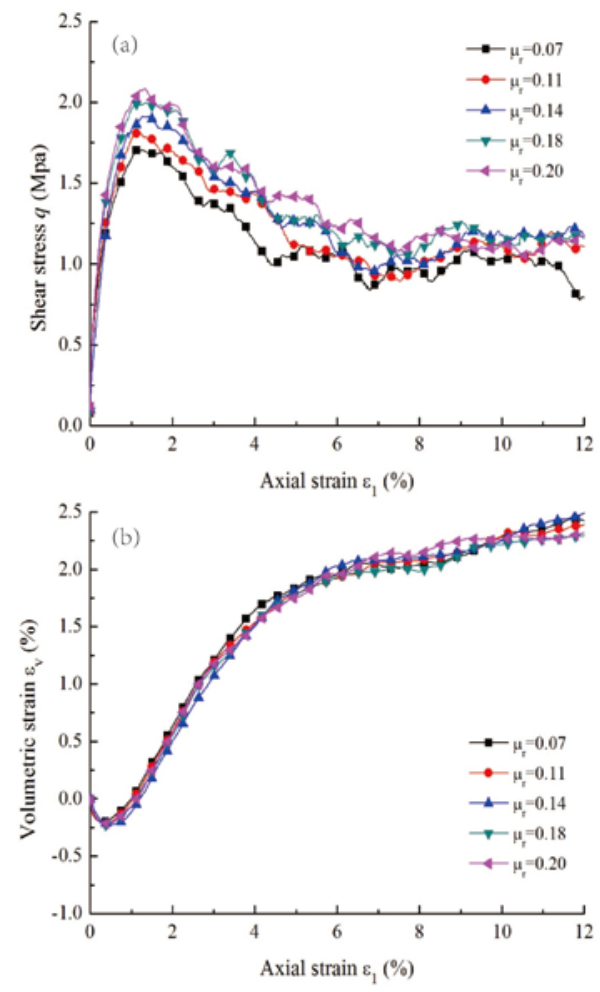

Fig. 4. Stress-strain relations for circular particles with varying rolling frictions in biaxial tests: (a) $q$ versus $\varepsilon_{l}$, and (b) $\varepsilon_{v}$ versus $\varepsilon_{l}$.

The macro-properties introduced by rolling resistance are somewhat different from the effects of particls shape, 
as above. The reason of these discrepancies is realatied to the increasing compressibility of samples as the particle shape becomes more elongated, which cannot be reproduced by increasing rolling friction.

Table 3. Comparison of the macro-parameters for the two sets of smaples.

\begin{tabular}{|c|c|c|c|c|c|c|}
\hline \multicolumn{2}{|c|}{$\delta\left(\mu_{r}\right)$} & $\begin{array}{c}0.49 \\
(0.07)\end{array}$ & $\begin{array}{c}0.79 \\
(0.11)\end{array}$ & $\begin{array}{c}1.10 \\
(0.14)\end{array}$ & $\begin{array}{c}1.45 \\
(0.18)\end{array}$ & $\begin{array}{c}1.76 \\
(0.20)\end{array}$ \\
\hline \multirow{2}{*}{$q_{\max }(\mathrm{Mpa})$} & $\mathrm{CP}$ & 1.54 & 1.79 & 2.02 & 2.07 & 2.09 \\
\cline { 2 - 7 } & $\mathrm{RL}$ & 1.71 & 1.81 & 1.94 & 2.02 & 2.08 \\
\hline \multirow{2}{*}{$q_{\text {res }}(\mathrm{Mpa})$} & $\mathrm{CP}$ & 1.16 & 1.21 & 1.69 & 1.87 & 1.84 \\
\cline { 2 - 7 } & $\mathrm{RL}$ & 1.03 & 1.1 & 1.18 & 1.13 & 1.13 \\
\hline \multirow{2}{*}{$E(\mathrm{Mpa})$} & $\mathrm{CP}$ & 140 & 150 & 148 & 137 & 138 \\
\cline { 2 - 7 } & $\mathrm{RL}$ & 470 & 497 & 470 & 529 & 550 \\
\hline \multirow{2}{*}{$v$} & $\mathrm{CP}$ & 0.3 & 0.31 & 0.27 & 0.24 & 0.13 \\
\cline { 2 - 7 } & $\mathrm{RL}$ & 0.18 & 0.14 & 0.21 & 0.13 & 0.16 \\
\hline \multirow{2}{*}{$\varepsilon_{\text {vmax }}(\%)$} & $\mathrm{CP}$ & 0.28 & 0.31 & 0.41 & 0.42 & 0.57 \\
\cline { 2 - 7 } & $\mathrm{RL}$ & 0.19 & 0.21 & 0.23 & 0.22 & 0.21 \\
\hline
\end{tabular}

\subsection{Non-coaxiality}

In the simple shear test, the direction of the major principal stress and strain rate will rotate during shearing process. Their orientation angle can be obtained easily by the following [6]

$$
\tan \theta_{\sigma}=2 \sigma_{x y} /\left(\sigma_{y}-\sigma_{x}\right), \tan \theta_{\Delta \varepsilon}=2 \Delta \varepsilon_{x y} /\left(\Delta \varepsilon_{y}-\Delta \varepsilon_{x}\right) .
$$
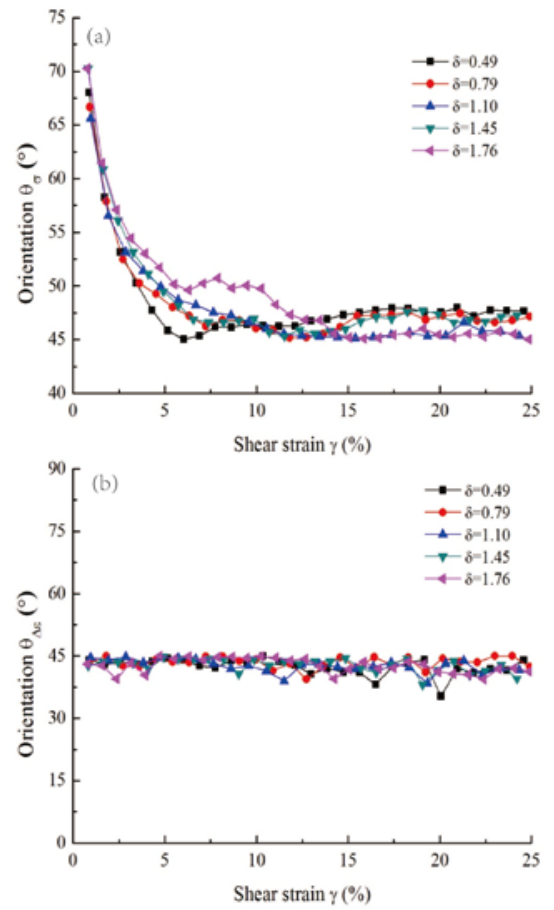

Fig. 5. Evolutions of (a) major principal stress directions, and (b) major principal strain rate directions for binary clumped particles of varying relative spacing in simple shear tests.

For the two sets of the samples, the evolutions of the major principal stress and strain rate directions (inclination to horizontal) are shown in the Figure 5 and 6 , respectively. It is noted that the a slower rate in approaching coaxiality is observed in the sample of clumped particles with a large relative spacing. However, the rolling friction has no obvious effect on the non- coaxiality before shear strain reached about 5\%. After $\gamma=5 \%$, the evolutions of the principal stress directions start to emerge differences, but the regularity is not obvious.
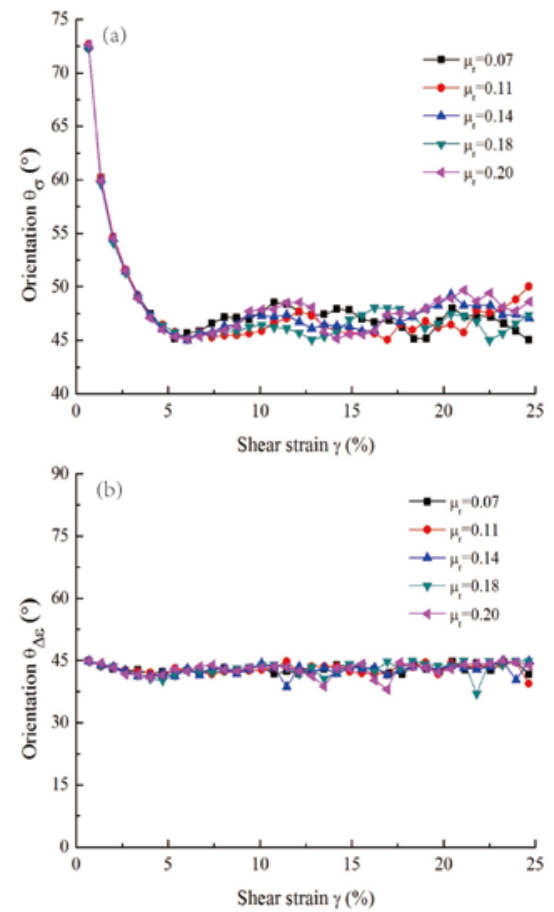

Fig. 6. Evolutions of (a) major principal stress directions, and (b) major principal strain rate directions for circular particles with varying rolling frictions in simple shear tests.

\section{Conclusion}

Two sets of samples, composed of binary clumped particles and circular particles with rolling resistance, are tested in DEM simulation. Rolling resistance reproduces well the effect of particle shape on the peak shear strength. However, different macro-properties, such as residual strength, elasticity modulus, poisson's ratio, dilatancy and non-coaxiality, introduced by rolling resistance are observed compared with particle shape. The discrepancies are assumed to be due to the fact that the increasing compressibility of samples, as the particle shape becomes more elongated, cannot be reproduced by increasing rolling friction.

\section{References}

1. N. Estrada, E. Azema, F. Radjai, A. Taboada. Rev. E 84, 011306 (2011)

2. C.M. Wensrich, A. Katterfeld, D. Sugo. Powder. Technol. 217, (2012)

3. K. Iwashita, M. Oda. J. ENG. MECH. ASCE. 124, (1998)

4. J. Ai, J.F. Chen, J.M. Rotter, J.Y. Ooi. Powder. Technol. 206, (2011)

5. Y.C. Zhou, B.D. Wright, R.Y. Yang, B.H. Xu, A.B. Yu. Physica. A. 269, (1999)

6. J. Ai, P.A. Langston, H.S. Yu. Int. J. Numer. Anal. Met. 38, (2014) 